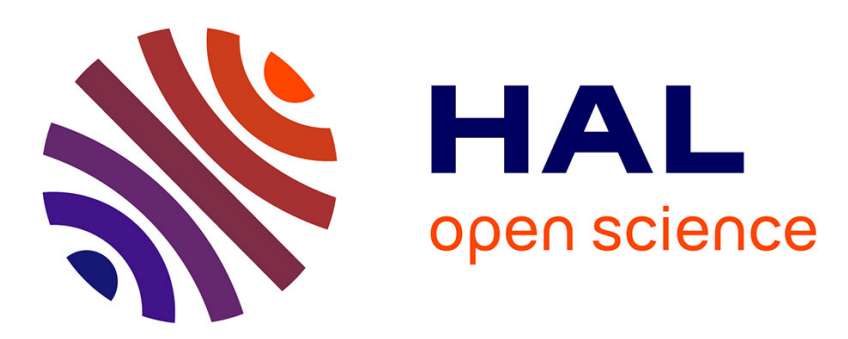

\title{
Characterization of the acoustic interactions in a two-staged multi-injection combustor fed with liquid fuel
} Theodore Providakis, Laurent Zimmer, Philippe Scouflaire, Sébastien Ducruix

\section{To cite this version:}

Theodore Providakis, Laurent Zimmer, Philippe Scouflaire, Sébastien Ducruix. Characterization of the acoustic interactions in a two-staged multi-injection combustor fed with liquid fuel. Journal of Engineering for Gas Turbines and Power, 2012, 134 (11), pp.111503. 10.1115/1.4007200 . hal00752290

\section{HAL Id: hal-00752290 \\ https://hal.science/hal-00752290}

Submitted on 15 Nov 2012

HAL is a multi-disciplinary open access archive for the deposit and dissemination of scientific research documents, whether they are published or not. The documents may come from teaching and research institutions in France or abroad, or from public or private research centers.
L'archive ouverte pluridisciplinaire HAL, est destinée au dépôt et à la diffusion de documents scientifiques de niveau recherche, publiés ou non, émanant des établissements d'enseignement et de recherche français ou étrangers, des laboratoires publics ou privés. 


\title{
Characterization of the Acoustic Interactions in a Two-Stage Multi-Injection Combustor Fed with Liquid Fuel
}

\author{
Theodore Providakis ${ }^{1,2}$ \\ ${ }^{1}$ CNRS, UPR 288 - Laboratoire EM2C, \\ 92290 Châtenay-Malabry, FRANCE \\ ${ }^{2}$ Ecole Centrale Paris, \\ 92290 Châtenay-Malabry, FRANCE \\ Email: theodore.providakis@ecp.fr
}

\author{
Laurent Zimmer ${ }^{1,2}$ \\ CNRS, UPR 288 - Laboratoire EM2C, \\ 92290 Châtenay-Malabry, FRANCE \\ ${ }^{2}$ Ecole Centrale Paris, \\ 92290 Châtenay-Malabry, FRANCE \\ Email: theodore.providakis@ecp.fr
}

\section{Philippe Scouflaire ${ }^{1,2}$}

${ }^{1}$ CNRS, UPR 288 - Laboratoire EM2C, 92290 Châtenay-Malabry, FRANCE

${ }^{2}$ Ecole Centrale Paris, 92290 Châtenay-Malabry, FRANCE Email: philippe.scouflaire@ecp.fr

\author{
Sébastien Ducruix ${ }^{1,2 *}$ \\ CNRS, UPR 288 - Laboratoire EM2C, \\ 92290 Châtenay-Malabry, FRANCE \\ ${ }^{2}$ Ecole Centrale Paris, \\ 92290 Châtenay-Malabry, FRANCE \\ Email: sebastien.ducruix@ecp.fr
}

\begin{abstract}
Burners operating in lean premixed prevaporized (LPP) regimes are considered as good candidates to reduce pollutant emissions from gas turbines. Lean combustion regimes result in lower burnt gas temperatures and therefore a reduction on the $\mathrm{NO}_{x}$ emissions, one of the main pollutant species. However, these burners usually show strong flame dynamics, making them prone to various stabilization problems (combustion instabilities, flashback, flame extinction). To face this issue, multi-injection staged combustion can be envisaged. Staging procedures enable fuel distribution control, while multipoint injections can lead to a fast and efficient mixing. A laboratory-scale staged multipoint combustor is developed in the present study, in the framework of LPP combustion, with an injection device close to the industrial one. Using a staging procedure between the primary pilot stage and the secondary multipoint one, droplet and velocity field distributions can be varied in the spray that is formed at the entrance of the combustion chamber. The resulting spray and the flame are characterized using OH-Planar Laser Induced Fluorescence, High Speed Particle Image Velocimetry and Phase Doppler Anemometry measurements. Three staging values, corresponding to three different flame stabilization processes, are analyzed, while power is kept constant. It is shown that mean values are strongly influenced by the fuel distribution and the flame position. Using adequate post-processing, the interaction between the acoustic field and the droplet behavior is characterized. Spectral analysis reveals a strong acoustic-flame coupling leading to a low frequency oscillation of both the velocity field and the spray droplet distribution. In addition, acoustic measurements in the feeding line show that a strong oscillation of the acoustic field leading to a change in fuel injection, and hence droplet behavior.
\end{abstract}

\section{Nomenclature \\ $f \quad$ frequency $[\mathrm{Hz}]$ \\ $\dot{m}$ mass flow rate $\left[\mathrm{g} \cdot \mathrm{s}^{-1}\right]$ \\ $\dot{Q} \quad$ volumic flow rate $\left[1 \cdot \mathrm{h}^{-1}\right]$}



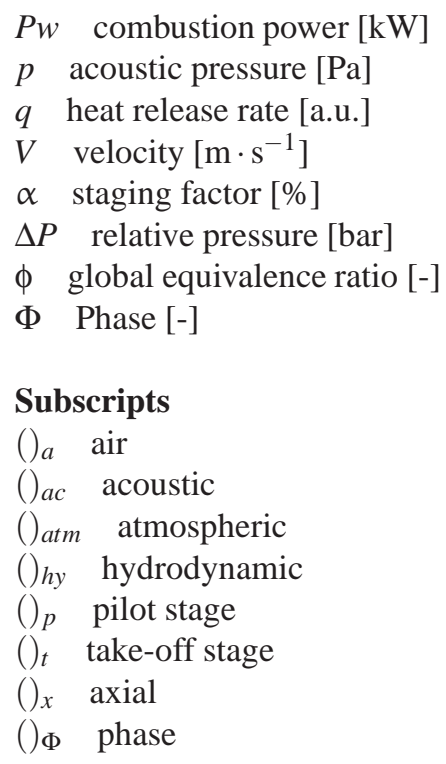

\title{
Superscripts
}

()$^{\prime} \quad$ fluctuations

()$^{\star}$ normalized

\author{
Acronyms \\ HSPIV High Speed Particle Image Velocimetry \\ LPP Lean Premixed Prevaporized \\ PDA Phase Doppler Anemometry \\ PLIF Planar Laser Induced Fluorescence \\ PSD Power Spectral Density \\ PVC Precessing Vortex Core
}

\section{INTRODUCTION}

Due to environmental concerns, permissible pollutant emissions of gas turbine plant or aircraft engines have been significantly decreased in recent years [1]. Combustion in gas turbines was traditionally based on non-premixed flames for various reasons (safety, stability), but this type of combustion leads to large pollutant emissions $\left(\mathrm{NO}_{\mathrm{x}}, \mathrm{CO}, \ldots\right)$. To face this issue, Lean Premixed Prevaporized (LPP) regimes are envisaged in new generation combustors [2, 3, 4]. This concept consists in providing a uniform lean mixture of fuel and air that burns at lower temperature than non-premixed flames, mainly reducing thermal $\mathrm{NO}_{\mathrm{x}}$ emissions. Unfortunately gas turbines operating in lean conditions often present high combustion dynamics, leading to stability issues such as combustion instabilities, flashback, self-ignition and blowout [5]. In particular, the coupling of heat release and pressure oscillations in the combustor can produce self-excited oscillations of such an amplitude that they may damage the combustor $[6,7,8]$. It is known that these acoustic interactions tend to develop more easily in partially and perfectly premixed combustion systems such as the LPP ones [9]. As an example of what can be envisaged to overcome those problems, secondary fuel injection has been proposed, for which a small amount of fuel is injected upstream to constitute a piloting region. This secondary injection can also be modulated so as to reduce coupling between heat release and pressure while keeping reduced $\mathrm{NO}_{\mathrm{x}}$ emissions [10, 11, 12, 13, 14].

Multi-injection staged injectors are now considered as potential candidates for real engine operations. Staging procedures enable fuel distribution control, while multipoint injections can lead to a fast and efficient mixing. However, the dynamics of these new generation injection devices must still be studied to clearly determine their stability properties and to optimize spatial fuel distribution.

The present paper concerns the study of a multi-injection system, fed with liquid fuel (dodecane) to be more representative of practical applications. Operating with a liquid fuel adds new critical parameters such as droplet distribution and evaporation, that strongly influence the flame dynamics. Depending on the type of atomizer used for fuel injection, strong fluctuations can be encountered in the resulting spray [15]. In the present study, a laboratory-scale staged multipoint combustor is developed in the framework of LPP combustion. Depending on the regime and staging factor, strong combustion instabilities can be encountered. Using the staging procedure between the primary and the secondary stages defined in [16], droplet and velocity field distributions can be varied in the spray that is formed at the entrance of the combustion chamber. The reactive flow is characterized using Phase Doppler Anemometry (PDA), High Speed Particle Image Velocimetry (HSPIV) and Planar Laser 
Induced Fluorescence (PLIF).Three staging values, corresponding to three different flame stabilization processes, are analyzed, while power is kept constant. Spray and flame dynamics are described using spectral post-processing. A synchronized phase-lock averaging procedure is finally proposed to go deeper in the analysis of this highly coupled dynamic system.

\section{EXPERIMENTAL SETUP}

The injection device is composed of two stages where air and liquid fuel can flow and mix. The resulting mixture enters a rectangular combustion chamber $\left(500 \times 150 \times 150 \mathrm{~mm}^{3}\right)$, composed of two silica windows for optical access and two water-cooled walls. The water flow rate is regulated so that the water exit temperature remains constant for all operating conditions.

\section{Injection device}

A schematic view of the injection device is shown in Fig. 1. Inside this device, the upstream (primary) stage is called the 'Pilot stage'. It is composed of a pressurized nozzle for fuel distribution and a swirler for air injection. The pressurized nozzle generates a solid cone and fuel can be injected at a maximum flow rate of 6.3 liters per hour. Its flow number is equal to $1.4 \mathrm{l} \cdot \mathrm{h}^{-1} \cdot \mathrm{bar}^{-0.5}$. The air swirler is composed of 18 vanes and it is geometrically designed so that $20 \%$ of the global air rate flows through this stage. This has been experimentally verified in [17]. The downstream (secondary) stage is called the 'Take-off stage'. It is composed of a multipoint system for the fuel and a swirler for the air. The multi-injection system is composed of 10 equally-spaced holes $(0.3 \mathrm{~mm}$ in diameter $)$. The swirler is composed of 20 vanes and it has been designed so that $80 \%$ of the global air rate flows through this stage. Both swirlers are set co-rotating (but this could be easily modified) and designed so that the swirl number $\mathrm{S}$ based on geometrical considerations is close to 1 [18]. To enhance fuel vaporization, air is preheated at $473 \mathrm{~K}$.

\section{Operating conditions}

As staging is one of the main features of this type of injection system, a staging factor $\alpha$ is defined to quantify the relative amount of fuel injected through the primary (pilot) injector [16]:

$$
\alpha=\frac{\dot{m}_{f, p}}{\dot{m}_{f, g}} \times 100
$$

where $\dot{m}_{f, g}$ is the total fuel flow rate and $\dot{m}_{f, p}$ is the fuel flow rate through the primary stage. As a consequence, $\alpha$ will be zero in case all fuel flows through the secondary (take-off) stage and $100 \%$ for all fuel injected through the pilot stage.

Table 1 shows the operating conditions chosen for the present study. These conditions were chosen to avoid flame extinction, which depends on both equivalence ratio and injection conditions. Indeed, for high staging values, the lean blowout was found for an equivalence ratio between 0.4 and 0.45 . However, decreasing the pilot injection (i.e. low staging values down to zero) can lead to flame extinction at high global equivalence ratios. The global air and fuel flow rates are kept constant (constant power and global equivalence ratio) while $\alpha$ is varied from 20 to $60 \%$, a domain where the shape of the flame is highly influenced by the staging procedure. Moreover, for a given $\alpha$, two types of flame can be encountered, whether $\alpha$ is varied from 20 to $60 \%$ or from 60 to $20 \%$. Subsequently, we define $\alpha^{+}$and $\alpha^{-}$, indicating that the corresponding value was obtained by increasing or decreasing the staging factor. For values of $\alpha^{-}$higher than $40 \%$ (pilot stage regimes), the flame stabilization process is controlled by the pilot stage, leading to a compact V-flame, anchored inside the injection device where the intense reaction takes place (Fig. 3). For values of $\alpha^{-}$lower than $25 \%$ (take-off stage regimes), the flame is stabilized thanks to the take-off stage and takes an M-shape. In this case, the main reaction occurs in the dump region (Fig. 3 ). In-between, there seems to be a competition between both stages, leading to a tulip-like shape of the flame. This latter case will not be discussed in the present study, where measurements focus on two extreme values of the staging factor (20 and $60 \%$ ), representative of the two flame shapes. Finally, for all values of $\alpha^{+}$, the flame presents an M-shape as for values of $\alpha^{-}$lower than $25 \%$. It must be noticed that combustion and hydrodynamic instabilities are encountered in both cases, associated with varying acoustic / hydrodynamic activities depending on the fuel staging. 
Table 1. OPERATING AND PRESSURE CONDITIONS. $P w=85 \mathrm{~kW}, \phi=0.6, P_{a}=101325 \mathrm{~Pa} . \Delta P_{a}$ IS THE PRESSURE DROP THROUGH THE INJECTION DEVICE.

\begin{tabular}{|c|cccccc|}
\hline Condition & $\dot{m}_{a}$ & $\begin{array}{c}\dot{Q}_{f, g} \\
{\left[\mathrm{~g} \cdot \mathrm{s}^{-1}\right]}\end{array}$ & $\alpha$ & $\Delta P_{f, t}$ & $\Delta P_{f, p}$ & $\Delta P_{a} / P_{a}$ \\
{$[\%]$} & {$[\mathrm{bar}]$} & {$[\mathrm{bar}]$} & {$[\%]$} \\
\hline $\mathrm{OP}_{20}$ & 50 & 9.4 & $\mathbf{2 0}$ & 0.13 & 1.9 & 5 \\
\hline $\mathrm{OP}_{60}^{+}$ & 50 & 9.4 & $\mathbf{6 0}^{+}$ & 0.08 & 18 & 5 \\
\hline $\mathrm{OP}_{60}^{-}$ & 50 & 9.4 & $\mathbf{6 0}^{-}$ & 0.08 & 18 & 5 \\
\hline
\end{tabular}

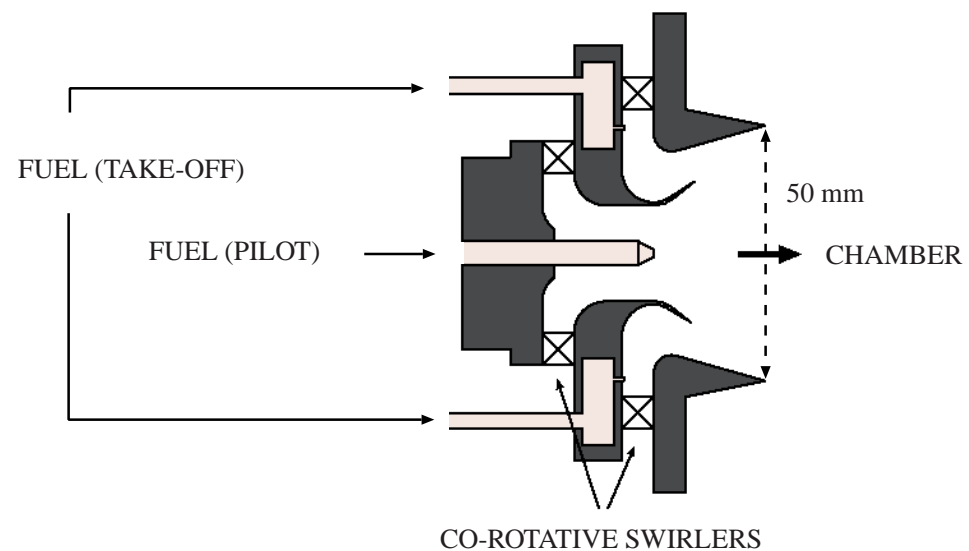

Fig. 1. SCHEMATIC VIEW OF THE INJECTION DEVICE. FLOW FROM LEFT TO RIGHT.

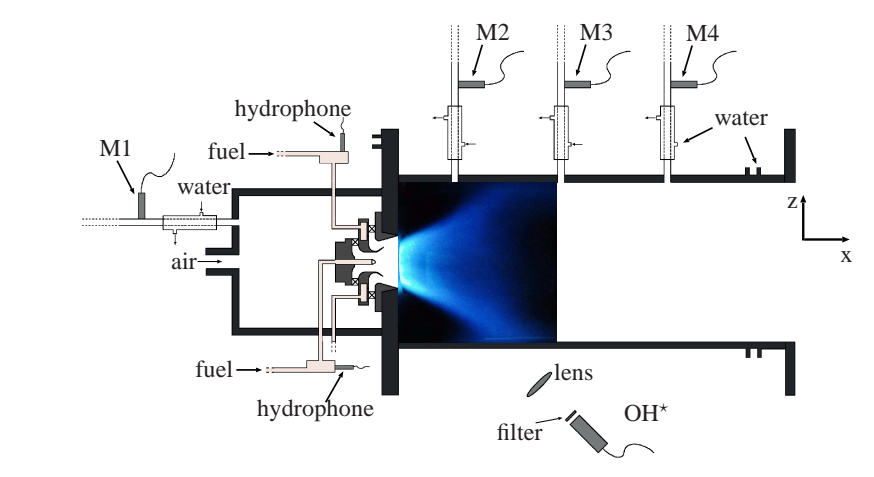

PRESSURE AND HEAT RELEASE MEASUREMENT (OP ${ }_{60}^{-}$FLAME IMAGE)

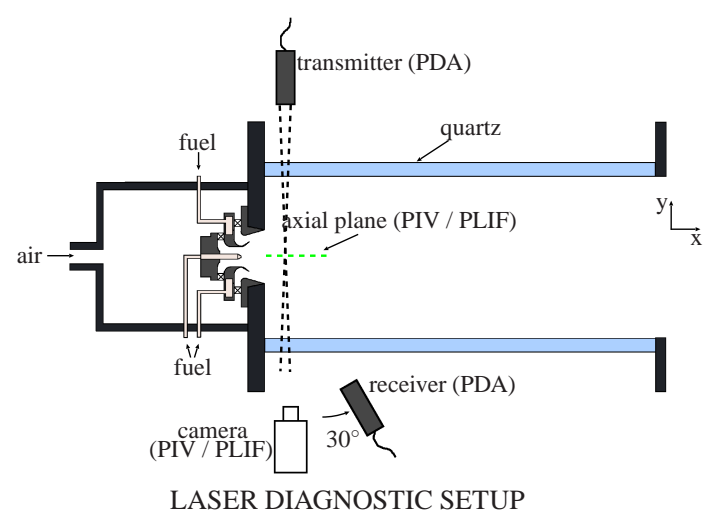

Fig. 2. EXPERIMENTAL SETUP. FLOW FROM LEFT TO RIGHT.

\section{Diagnostics}

Spray characterization

The spray is characterized using High Speed Particle Image Velocimetry (HSPIV) in the axial direction and Phase Doppler Anemometry (PDA) measurements. It was decided to use fuel droplets directly as tracers. The camera collects the Mie scattering signal from individual droplets cut by the laser sheet. It is assumed that, at the exit of the injection system, droplets are small enough to describe the aerodynamic flow. This point will be discussed in the later analysis.

The laser sheet is generated by a system consisting of two Nd:YAG lasers (Quantronix). Both lasers emit pulses at a wavelength of $532 \mathrm{~nm}$ with an energy of $5 \mathrm{~mJ}$ per pulse and a temporal width of $120 \mathrm{~ns}$. An optical system (Melles Griot) is used to convert the laser beam into a planar light sheet $100 \mathrm{~mm}$ wide and $1 \mathrm{~mm}$ thick. Both sides of the combustion chamber contain rectangular silica windows. For the axial measurements, two small rectangular quartz windows (100 mm long and $15 \mathrm{~mm}$ wide) were designed and placed in the upper and lower walls of the combustion chamber, allowing the laser sheet to 
cross the chamber in its axial direction.

A fast speed camera (Photron Fastcam SA5, $1024 \times 1024$ pixels at a rate of 6000 frames per second) equipped with a $105 \mathrm{~mm}$ F/2.8 Nikon Nikkor objective is used to acquire the resulting images. The two lasers work at half the camera's acquisition frequency, $\mathrm{f}_{\mathrm{cam}}$, and are synchronized by a pulse delay generator (BNC 555 pulse/delay Generator). The time delay, $\delta \mathrm{t}$, between two pulses has been chosen so that the displacement of a droplet does not exceed one fourth of the processing window size (4 pixels in this study). The acquisition parameters are summarized in Table 2 and a schematic view of the setup is presented in Fig. 2.

For the local characterization of the spray, a Dantec dual-beam PDA system is installed in the present configuration allowing to measure the local distributions of droplet diameters and two velocity components. The system is composed of a $5 \mathrm{~W}$ $\mathrm{Ar}^{+}$laser with two lines respectively at $514 \mathrm{~nm}$ and $488 \mathrm{~nm}$ combined with a $40 \mathrm{MHz}$ Bragg cell for frequency shift. Two lenses are used for the transmitting and receiving optics with respective focal lengths at $250 \mathrm{~mm}$ and $300 \mathrm{~mm}$. The receiver is placed at $30^{\circ}$ from the transmitter axis. Data are acquired at different locations using an automatic two-axis translation system allowing high precision on the measurement location. Laser beam intersections are kept at the center of the chamber along the $Y$ axis position, while location is varied on the $X$ and $Z$ axis.

Data rates between 0.2 and $12 \mathrm{kHz}$ and a minimum burst efficiency of 0.6 could be achieved. During tests, more than 100,000 samples are validated in the main regions of interest, whereas in low signal regions, the acquisition time is limited to 30 seconds, resulting on lower data rates.

Table 2.
\begin{tabular}{|cc|}
\hline $\mathrm{f}_{\mathrm{cam}}[\mathrm{fps}$ ] & 20,000 \\
\hline$\delta \mathrm{t}[\mu \mathrm{s}$ ] & 6 \\
\hline Nb of raw images [ - ] & 12,000 \\
\hline Image size [ px ] & $776 \times 448$ \\
\hline Camera pitch [ mm / px ] & 0.15 \\
\hline
\end{tabular}

\section{Flame structure characterization}

The reaction zone is characterized using Planar Laser Induced Fluorescence (PLIF) measurements in axial planes. The PLIF technique is carried out by exciting the $\mathrm{OH}$ radical, which is a good tracer to describe the structure of the flame. The laser sheet is generated by a system consisting of a Nd:YAG laser (Continuum Powerlite DLS 8010 series), emitting a pulse at a wavelength of $532 \mathrm{~nm}$, coupled to a Dye Laser (Continuum ND6000). The dye laser contains a UV tracker that doubles the laser output to cover the $206 \mathrm{~nm}$ and $425 \mathrm{~nm}$ wavelength ranges. A cylindrical divergent and a spherical converging lenses with respective focal lengths at $250 \mathrm{~mm}$ and $300 \mathrm{~mm}$ are used to generate a laser sheet $70 \mathrm{~mm}$ wide and $1 \mathrm{~mm}$ thick. The $\mathrm{OH}$ radical can be excited at different wavelengths but the emitted intensity changes. For this study, the maximum intensity was detected for a wavelength $\lambda=282.9 \mathrm{~nm}$ (probably the $Q_{1}(5)$ transition of the $(1,0)$ band of the $A^{2} \Sigma^{+} \rightarrow X^{2} \Pi$ system of $\mathrm{OH}[18])$.

Table 3. OH-PLIF ACQUISITION PARAMETERS
\begin{tabular}{|cc|}
\hline $\mathrm{f}_{\mathrm{cam}}[\mathrm{fps}]$ & 10 \\
\hline Exposure time [ $\mathrm{ns}]$ & 100 \\
\hline Nb of raw images [ - ] & 10,000 \\
\hline Image size [ px ] & $512 \times 512$ \\
\hline$\lambda$ [ nm ] & 282.9 \\
\hline Camera pitch [ mm / px ] & 0.15 \\
\hline
\end{tabular}


Images are acquired using an Intensified CCD camera (Princeton Instruments PI-MAP 3, $1024 \times 1024$ pixels at a rate of 15 images per second) equipped with a $105 \mathrm{~mm}$ F/4.5 Nikon Nikkor UV objective coupled to two interferential filters (Melles Griot, WG 305 and UG 11) centered on the $\mathrm{OH}$ radical emission band. Measurements are performed at $10 \mathrm{~Hz}$ and the acquisition parameters are summarized in Table 3.

\section{Pressure and heat release fluctuations}

Four Bruel \& Kjaer microphones (M1, M2, M3 and M4) are placed in semi-infinite water cooled waveguides that are flush-mounted close to the injection device and on the combustion chamber axis to measure pressure fluctuations (Fig.2). A photomultiplier (Hamamatsu, H5784-04), coupled with a filter $(\lambda=310 \pm 10 \mathrm{~nm})$ and a spherical lens (focal = $300 \mathrm{~mm})$ to collect all the light emitted by the flame, is used to measure $\mathrm{OH}^{*}$ intensity fluctuations. This last signal is supposed to be proportional to the heat release rate for premixed flames [19], giving access to heat release fluctuations, a crucial quantity in the understanding of combustion instabilities. In the case of partially premixed flames, several studies [20, 21] have shown that the $\mathrm{OH}^{*}$ emission still provides qualitative information on the heat release fluctuations, on a global point of view.

All signals are acquired simultaneously on a multi-port acquisition card (National Instruments), at a rate of $16 \mathrm{kHz}$ during 4 seconds for the HSPIV measurements and 60 seconds for the PLIF ones.

\section{RESULTS}

\section{Flame stabilization}
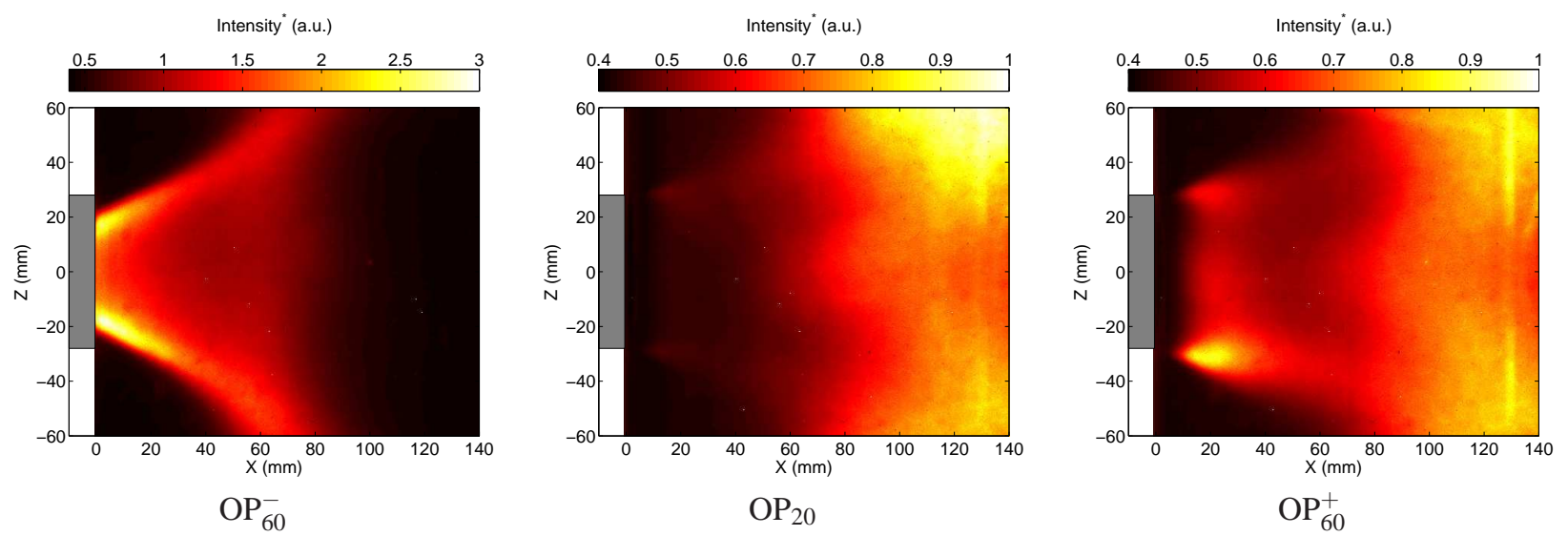

Fig. 3. AVERAGED OH CHEMILUMINESCENCE FOR THE THREE OPERATING POINTS. INTENSITY SCALE HAS BEEN NORMALIZED BY THE MAXIMUM OT THE OP 20 INTENSITY VALUE. FLOW FROM LEFT TO RIGHT.

Figure 3 shows the average $\mathrm{OH}^{\star}$ chemiluminescence for the three operating points. When operating at high staging factor, $\mathrm{OP}_{60}^{-}$, the flame stabilization is mainly controlled by the pilot stage resulting in a V-flame shape. This shape is encountered for staging values higher than $40 \%$. When decreasing this factor, the amount of fuel delivered through the pilot stage becomes insufficient to keep the flame stabilized close to the pilot stage, resulting in a M-shape flame stabilized in the combustion chamber. In this case, the flame is mainly controlled by the take-off stage and the flame is anchored at the exit of the injection system $\left(\mathrm{OP}_{20}\right)$. From the $\mathrm{OH}^{\star}$ averaged fields, it seems that the major combustion process takes place far downstream in the combustion chamber but a small reaction rate is found close to the injection device. When fuel is no longer injected through the pilot stage $(\alpha=0 \%)$, the flame becomes very unstable and is quickly extinguished. Finally, increasing the staging factor while the flame is stabilized in the combustion chamber $\left(\mathrm{OP}_{60}^{+}\right)$results in a shape close to the $\mathrm{OP}_{20}$ one. This is the result of an hysteresis (bifurcation) phenomenon and the flame does not return to its initial shape $\mathrm{OP}_{60}^{-}$. Although both $\mathrm{OP}_{20}$ and $\mathrm{OP}_{60}^{-}$present similar flame shapes, the combustion process is highly modified. Indeed the latter case shows a larger and more intense reaction region close to the injection device while downstream the opposite behavior is visible. Increasing alpha enhances the anchoring of the flame at the exit of the burner and improves its stability.

Recent work [22] has showed that the combustion structure is highly influenced by an aerodynamic instability (precessing vortex core, PVC), with a varying intensity depending on the injection conditions and acoustic levels. It is shown that for the V-flame, the PVC is very strong while for the M-shaped one, when acoustic levels are high, the PVC barely exists. It seems then that the pilot V-flame is driven by the PVC structure, while for the M-shaped one, the combustion structure may 
be the result of the strong thermo-acoustic coupling. Since the present paper focusses on the acoustic characterization of the combustor, the PVC/flame interactions will not be discussed here.

\section{Velocity fields}
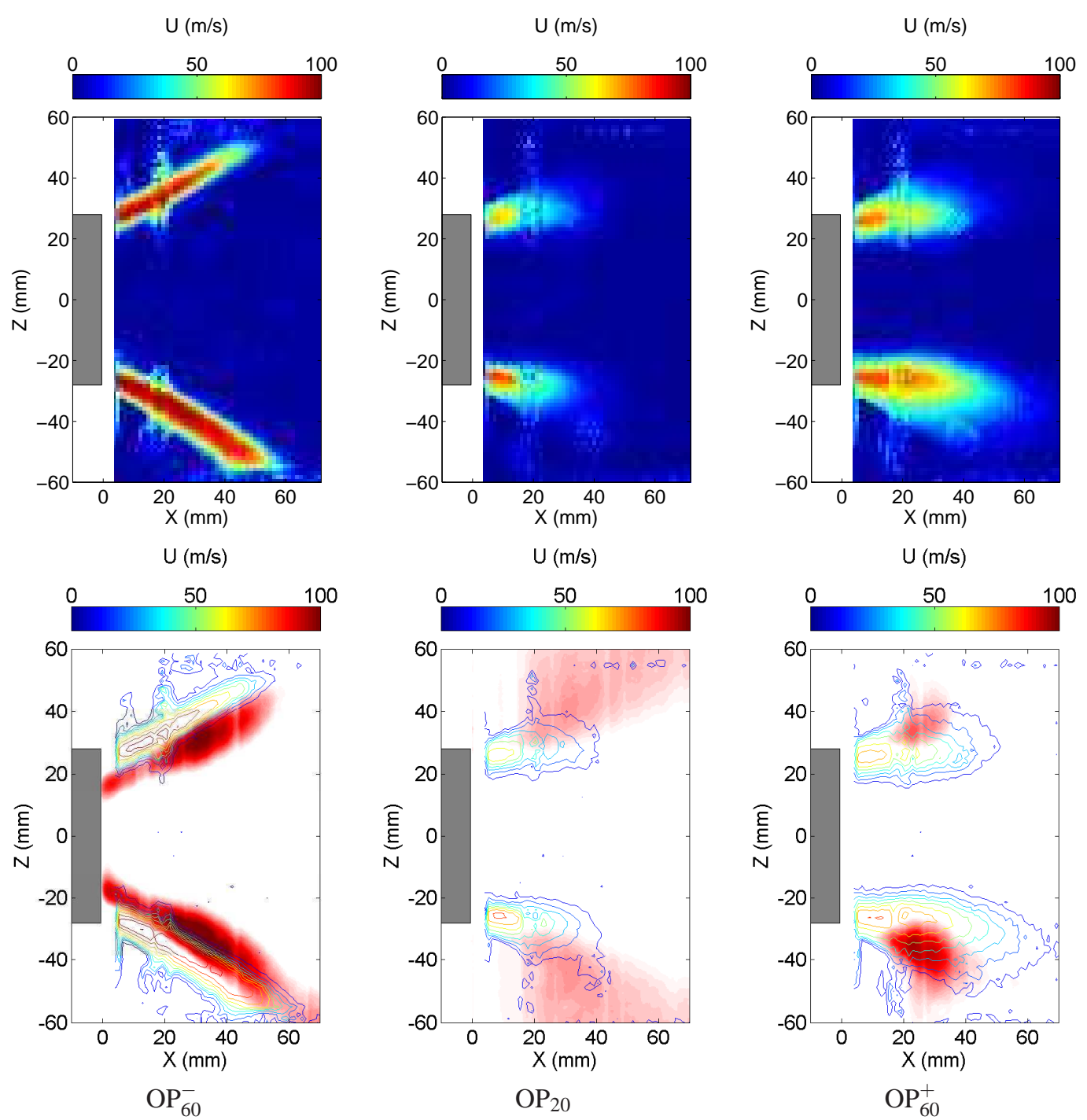

Fig. 4. AVERAGE DROPLET VELOCITY MAGNITUDE (TOP) AND OH-PLIF (BOTTOM) FIELDS FOR THE THREE OPERATING CONDITIONS. CONTOUR LINES OF THE VELOCITY MAGNITUDE ARE SUPERIMPOSED ON THE OH FIELDS. FLOW FROM LEFT TO RIGHT.

Figure 4 shows the average droplet velocity magnitude and OH-PLIF axial fields for the three operating points. The velocity field provides a quantitive information on the droplet velocity but also a qualitative information on the spray shape. For the $\mathrm{OP}_{60}^{-}$case, the spray has a wide angle while for $\mathrm{OP}_{20}$ and $\mathrm{OP}_{60}^{+}$the angle is lower. Also, in these latter cases, the length of the spray is lower than for $\mathrm{OP}_{60}^{-}$. This is the result of the flame behavior as it has been shown in [23] that the spray shape in non reactive conditions is not affected by the fuel distribution. It has also been found in [24] that $\mathrm{OP}_{20}$ generates lower size droplets at the exit of the injection device, thus resulting on a shorter spray. From the PIV fields, the OP 60 case presents faster droplets with up to $100 \mathrm{~m} \cdot \mathrm{s}^{-1}$ in the center region of the spray, corresponding also to the region where droplets of lower size are found. Although the $\mathrm{OP}_{60}^{+}$case presents the same fuel staging, lower velocities are achieved (up to $\left.80 \mathrm{~m} \cdot \mathrm{s}^{-1}\right)$. This is the result of the two different stabilization processes. In the first case, the flame is well stabilized in the pilot region inside the injection device while in the second one, it is anchored at the exit of the injection system. This results in a larger section area for the spray, thus leading to lower velocities for both $\mathrm{OP}_{20}$ and $\mathrm{OP}_{60}^{+}$. 


\section{Combustion process}

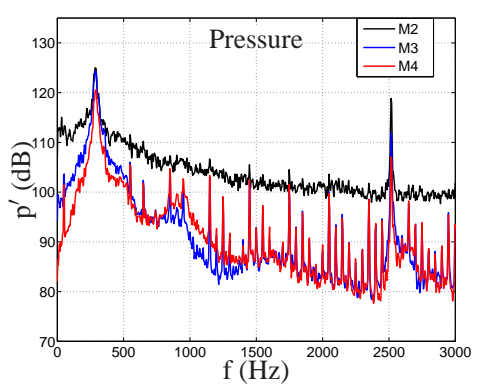

Pressure

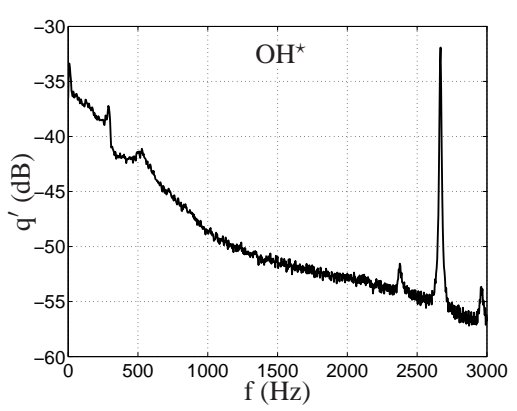

Heat release

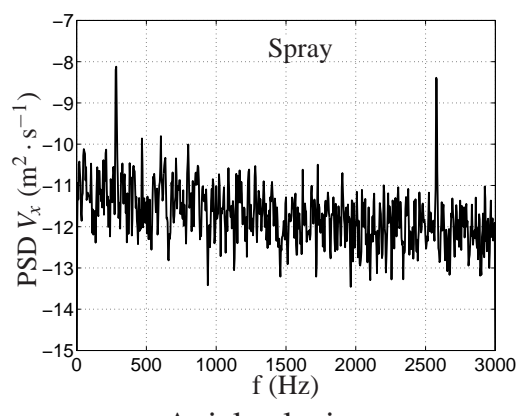

Axial velocity

Fig. 5. PSD OF THE ACOUSTIC PRESSURE (FROM MICROPHONE M2), HEAT RELEASE RATE (OH ${ }^{\star}$ CHEMILUMINESCENCE) AND DROPLET AXIAL VELOCITY FOR OP 60 CASE.

From the OH-PLIF averaged fields coupled to the PIV ones (Fig. 4), it is found that the interaction between droplets and flame is highly influenced by the staging factor and flame position. For the $\mathrm{OP}_{60}^{-}$case, the majority of the spray surrounds the flame and the reaction process occurs in the inner region of the spray. Meanwhile, as mentioned earlier, for the $\mathrm{OP}_{20}$ case, the main reaction process takes place far downstream in the combustion chamber even though smaller droplets are found in this case. However, analyzing the PIV fields, it is found that the generated spray is much larger in comparison to the $\mathrm{OP}_{60}^{-}$case and, since the flame is farther downstream, temperatures close to the injection device are lower and droplets take more time to evaporate. When increasing the staging factor, while keeping the flame in the combustion chamber, a more intense reaction zone takes place near the injection device $\left(\mathrm{OP}_{60}^{+}\right)$. This can be explained by two different processes. (1) It was verified that augmenting the fuel flowrate through the pressurized nozzle results in a decrease of the size of the generated droplets. Therefore, a faster evaporation time is achieved and the reaction process happens sooner. (2) Decreasing the amount of fuel through the takeoff stage enhances the atomization of the liquid jets and decreases the size of the droplets at the exit of the injection system [25]. Both situations will contribute to a faster reaction close to the injection system.

\section{Unsteady flame dynamics}

To characterize the dynamic behavior of the burner, a spectral analysis is performed on the pressure, heat release and axial velocity (acquired through the PDA system at $\mathrm{X}=15 \mathrm{~mm}$ ) signals. For each signal, a PSD is computed using the Welch method, with a spectral resolution of $4 \mathrm{~Hz}$. Let us notice that PDA signals have been re-sampled at $6 \mathrm{kHz}$ as described in [24]. Figure 5 successively shows the PSD of the heat release rate $\left(\mathrm{OH}^{*}\right.$ chemiluminescence), acoustic pressure (from microphone $\mathrm{M} 2$ ), and axial velocity for the $\mathrm{OP}_{60}^{-}$case.

The acoustic pressure reveals a strong peak centered at $f_{a c}=300 \mathrm{~Hz}$, which is also seen by the $\mathrm{OH}^{\star}$ spontaneous emission, both in phase as expected by the Rayleigh criterion in the case of thermo-acoustic instabilities. Droplet velocities also present a strong activity at the same frequency $f_{a c}$, revealing that this instability modifies the spray dynamics. More interestingly, a second peak centered at $f_{h y} \approx 2600 \mathrm{~Hz}$ with a higher amplitude appears in the signal of heat release and axial velocity fluctuations. It has been shown that this frequency corresponds to the presence of a precessing vortex core (PVC) in both non-reactive and reactive conditions [23, 24]. Measurements in both conditions have shown that the PVC intensity is amplified in presence of the flame, with an increase of the detected frequency, probably due to higher temperature, hence velocities. Finally, the $\mathrm{OH}^{\star}$ signal reveals two additional peaks with very low amplitudes around the peak of the PVC frequency, at $f \approx 2300 \mathrm{~Hz}$ and $f \approx 2900 \mathrm{~Hz}$. This phenomenon has already been found in [26, 27] and recently analyzed in [28], where it is shown that these peaks correspond to a coupling phenomenon between the acoustics and the PVC. The detected frequencies correspond exactly to the sum and the difference of the acoustic and PVC ones.

To go deeper in the acoustic characterization of the system, two hydrophones have been placed in each fuel feeding line far upstream of the injection device. Figure 6 shows the evolution of the PSD amplitude in the combustion chamber and in the multipoint feeding line. No results are presented for the hydrophone placed in the pilot fuel feeding line as no acoustic peak was detected. This is no surprise as pressure drop values are high for the pilot injection as shown in table 1 . However, the hydrophone placed in the multipoint feeding line reveals a peak at the acoustic frequency detected in the combustion 
chamber. The fact that acoustic peaks are detected in this line comes from the low pressure drop values encountered in this stage (cf. table 1). The amplitude presents an evolution similar to the one measured in the combustion chamber. This clearly shows that the acoustic field strongly modifies the multipoint injection and consequently, evaporation and equivalence ratio may also be modified affecting the combustion process. This equivalence ratio fluctuation may also drive the instability corresponding to a potential coupling path [9]. Further investigation is being carried out on this subject. Globally, results indicate that for the $\mathrm{V}$-flame shape $\left(\alpha^{-}>40 \%\right)$, the acoustic perturbation is quite low in comparison to the M-shape one $\left(\alpha^{-}<40 \%\right.$ and for all $\alpha^{+}$values) where amplitudes of up to $145 \mathrm{~dB}$ are encountered. In addition, the change in the flame shape also results in a change in the acoustic frequency, with an increase from $f_{a c}=300 \mathrm{~Hz}$ to $f_{a c}=330 \mathrm{~Hz}$.
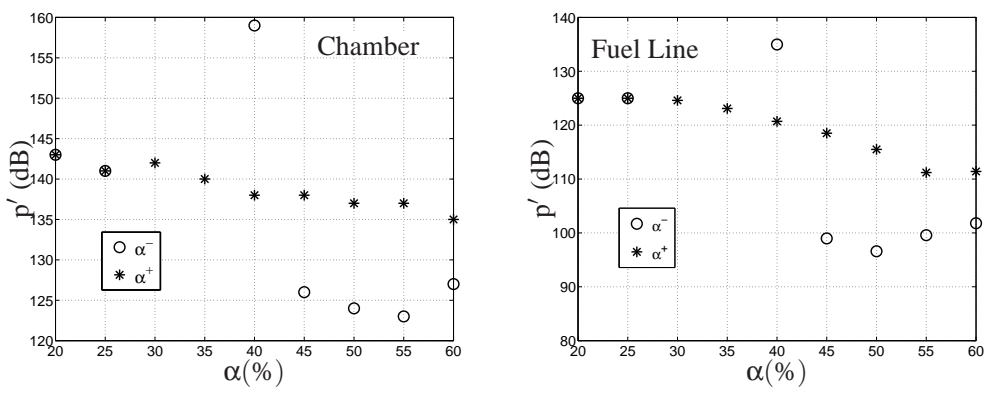

Fig. 6. EVOLUTION OF THE PSD AMPLITUDE FOR THE ACOUSTIC INSTABILITY IN THE COMBUSTION CHAMBER (LEFT) AND IN THE MULTIPOINT FEEDING LINE (RIGHT) AS A FUNCTION OF THE STAGING FACTOR.

To characterize the interaction between the acoustic field and the droplet dynamics, a phase-locked averaging method is performed, focussing on the three operating conditions defined in table 1 . This can only be possible if the droplets are small enough to be considered as following the aerodynamic flow. Therefore, an estimation of the Stokes number (with respect to the acoustic instability) has been computed and it was found that this number is close to 0.1 for a droplet size equal to $15 \mu \mathrm{m}$. Since the PDF of the droplets size distribution reported in [24] reveals that the generated spray is composed mainly of droplets below $15 \mu \mathrm{m}$, one may expect them to follow the aerodynamic flow. The phase-lock average cycle is computed at the acoustic frequency, $f_{a c}=300 \mathrm{~Hz}$ for the $\mathrm{OP}_{60}^{-}$and $f_{a c}=330 \mathrm{~Hz}$ for the $\mathrm{OP}_{60}^{+}$and $\mathrm{OP}_{20}$. It has already been found in a previous study [24], that droplet diameter fluctuations are barely affected by the acoustic field (variations due to fluctuations are below $1 \mu \mathrm{m}$ ), thus we will focus only on the droplet axial velocity fluctuations. The signal from microphone M2 is used as the time reference signal and the phase-locked mean cycle is decomposed in 20 phases, every $18^{\circ} \pm 9^{\circ}$, using approximately 2,000 velocity samples for each phase. Results are presented in Fig. 7.

As expected, results for the acoustic pressure indicate higher fluctuations (up to $400 \mathrm{~Pa}$ ) for the $\mathrm{OP}_{60}^{+}$and $\mathrm{OP}_{20} \mathrm{Cases}$ while for the $\mathrm{OP}_{60}^{-}$, pressure fluctuations do not exceed $100 \mathrm{~Pa}$. More interestingly, velocity fluctuations are not in phase as it would be expected. Several studies on the interaction of a spray with an acoustic field [29, 30, 31] have shown that when located at an acoustic velocity antinode (hence pressure node), the spray velocity field decreases in magnitude. The results obtained in the $\mathrm{OP}_{60}^{-}$case are consistent with this conclusion. However, the axial velocity for the two other cases presents a slight phase shift. It is well known that the presence of a phase shift can be related to the droplet size [29, 30, 32], since bigger droplets will need more time to follow the acoustic motion. Nevertheless, PDA measurements have shown that the spray in the $\mathrm{OP}_{60}^{+}$ and $\mathrm{OP}_{20}$ cases presents smaller droplets so this cannot be the cause of the phase shift. Furthermore, measurements carried out with the hydrophone indicate a stronger acoustic activity in the multi-injection line for the M-shape flame. Therefore, the phase shift may come from this perturbation which results in a change of the injection behavior, hence spray and mixture properties. Finally, trying to estimate the acoustic velocity amplitude using the simplistic relation $u_{a c}^{\prime} \approx p^{\prime} / \rho_{a} c$, which is only valid for plane wave progressive propagation, one finds $u_{a c}^{\prime} \approx 0.4 \mathrm{~m} \cdot \mathrm{s}^{-1}$ for the V-flame and $u_{a c}^{\prime} \approx 1.6 \mathrm{~m} \cdot \mathrm{s}$ for the M-flame. The measured fluctuations are consistent for the M-flame while for the V-flame, fluctuations are found to be 3 to 4 times higher. One suggestion could be that the acoustic field modulates the flow and the nascent spray inside the injector, resulting in large coherent structures that are generated at the acoustic frequency and convected by the flow $[33,34,35,36]$. A second suggestion could be that for the V-flame, the spray is strongly affected by the presence of the precessing vortex core (PVC) and fluctuations of up to $15 \%$ of the mean velocity have been found from the PDA results and PIV fields. In addition, a recent work [27] shows that, even when the acoustic coupling occurs with a much lower amplitude compared to the hydrodynamic phenomenon, some precautions must be taken while describing the flow behavior at one frequency and neglecting the other. 


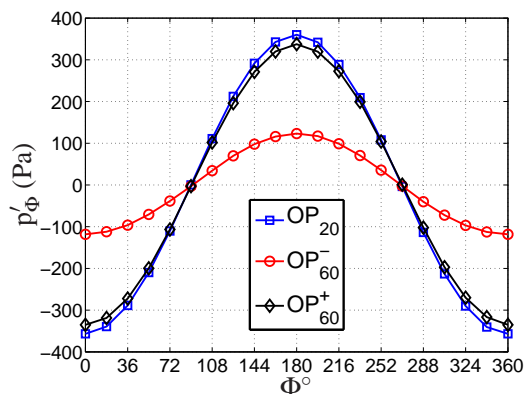

Pressure

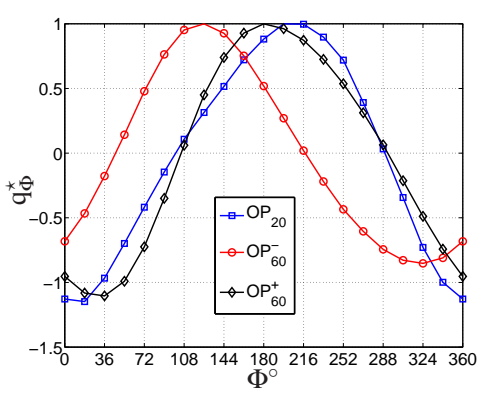

Heat release

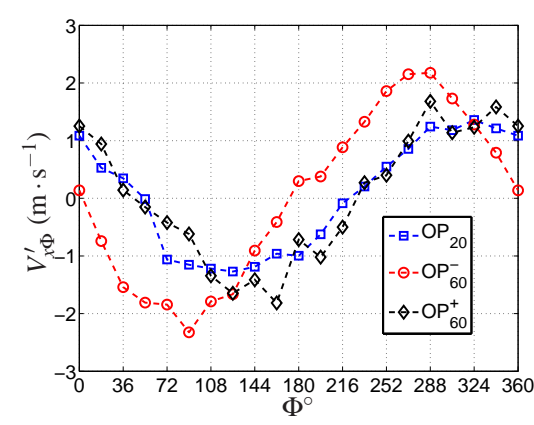

Axial Velocity

Fig. 7. FLUCTUATIONS OF THE PHASE AVERAGED ACOUSTIC PRESSURE $p_{\Phi}^{\prime}$, HEAT RELEASE RATE $q_{\Phi}^{\star}$, AND AXIAL VELOCITY $V_{x \Phi}^{\prime} \cdot \mathrm{OP}_{60}^{-}, f_{a c}=300 \mathrm{~Hz}$ AND OP ${ }_{60}^{+}$AND OP $_{20}, f_{a c}=330 \mathrm{~Hz}$.

\section{CONCLUSION}

A laboratory-scale staged multi-injection combustor is described in the present paper, in the framework of LPP combustion. Using a staging procedure between the primary pilot stage and the secondary multipoint one, droplet and velocity field distributions can be varied in the spray that is formed at the entrance of the combustion chamber. In the reactive case, three different stabilization processes occur, depending on the staging factor value and evolution history (hysteresis). Three staging values, corresponding to these three different flame stabilization processes, are analyzed while power is kept constant. The resulting spray and flame are characterized using OH-Planar Laser Induced Fluorescence, High Speed Particle Image Velocimetry and Phase Doppler Anemometry measurements.

It is first shown that the global behavior of the burner is highly influenced by the fuel distribution. When a sufficient amount of fuel is delivered through the pilot stage, the flame shows a V-shape and is stabilized inside the injection system. Decreasing the staging factor, the flame is stabilized in the combustion chamber and takes an M-shape. Switching off the pilot injection results in flame extinction. More interestingly, increasing back the staging factor value results in an hysteresis phenomenon, where the flame remains in the combustion chamber, even though the amount of fuel in the pilot region increases. This leads to a situation where for the same fuel distribution, two types of flame are encountered $\left(\mathrm{OP}_{60}^{-}\right.$and $\left.\mathrm{OP}_{60}^{+}\right)$.

The dynamic analysis reveals the presence of an acoustic-flame coupling leading to a low frequency oscillation of both the velocity field and the spray droplet distribution at $f_{a c}=300 \mathrm{~Hz}$ and $f_{a c}=330 \mathrm{~Hz}$ depending on the fuel distribution. Furthermore, acoustic measurements in the multi-injection feeding line reveal large acoustic fluctuations that evolve along with the acoustic amplitude in the combustion chamber. Using adequate post processing methods, the droplet response to the acoustic field has been characterized. For the $\mathrm{OP}_{60}^{-}$case, results are consistent with several studies on the behavior of droplets subjected to acoustic perturbations. However, for the $\mathrm{M}$-shape case $\left(\mathrm{OP}_{20}\right.$ and $\left.\mathrm{OP}_{60}^{+}\right)$, the velocity fluctuations present a slight phase shift that is likely to be the result of the acoustic perturbation inside the take-off injection, which modifies the spray properties (atomization and evaporation). This last point is currently under investigation.

\section{ACKNOWLEDGMENTS}

This work is supported by Pôle ASTECH and funded by Conseil Régional d'Ile-de-France (CRIF), in the framework of the INCA initiative. The authors would like to thank the technical staff at the EM2C laboratory for their help in mounting and designing the experimental setup.

\section{References}

[1] Correa, S., 1998. "Power generation and aeropropulsion gas turbines: From combustion science to combustion technology". Proceedings of the Combustion Institute, 27, pp. 1793-1807.

[2] Tacina, R., 1990. "Low NOx potential of gas turbine engines". AIAA Aerospace Sciences Meeting and Exhibit(900550), January.

[3] Lefebvre, A., 1995. "The role of fuel preparation in low-emission combustion". Journal of Engineering for Gas Turbines and Power, 117, pp. 617-654.

[4] Moore, M., 1997. "NOx emission control in gas turbines for combined cycles cycle gas turbine plant". Journal of Power and Energy, 211(1), pp. 43-52.

[5] Lieuwen, T., and Yang, V., 2006. Combustion Instabilities in Gas Turbine Engines: Operational Experience, Fundamental Mechanisms, and Modeling, Vol. 210. Progress in Astronautics and Aeronautics. 
[6] Candel, S., 2002. "Combustion dynamics and control progress and challenges". Proceedings of the Combustion Institute, 29, pp. 1-28.

[7] Nauert, A., Petersson, P., Linne, M., and Dreizler, A., 2007. "Experimental analysis of flashback in lean premixed swirling flames: conditions close to flashback". Experiments in Fluids, 43, pp. 89-100.

[8] Lefebvre, A., 1999. Gas Turbine Combustion, 2nd ed. Taylor \& Francis.

[9] Ducruix, S., Schuller, T., Durox, D., and Candel, S., 2003. "Combustion dynamics and instabilities: elementary coupling and driving mechanisms". Journal of Propulsion and Power, 19(5), Sep-Oct, pp. 722-734.

[10] Neumeier, Y., and Zinn, B. T., 1996. "Experimental demonstration of active control of combustion instabilities using real-time modes observation and secondary fuel injection”. Symposium (International) on Combustion, 26(2), pp. 2811-2818.

[11] Lee, J., Kim, K., and Santavicca, D., 2000. "Effect of injection location on the effectiveness of an active control system using secondary fuel injection". Proceedings of the Combustion Institute, 28, pp. 739-746.

[12] Bernier, D., Ducruix, S., Lacas, F., Candel, S., Robart, N., and Poinsot, T., 2003. "Transfer function measurements in a model combustor: Application to adaptive instability control”. Combustion Science and Technology, 175, pp. 9931013.

[13] Tachibana, S., Zimmer, L., Kurosawa, Y., and Suzuki, K., 2007. "Active control of combustion oscillations in a lean premixed combustor by secondary fuel injection coupling with chemiluminescence imaging technique". Proceedings of the Combustion Institute, 31(2), pp. 3225-3233.

[14] Barbosa, S., de La Cruz Garcia, M., Ducruix, S., Labegorre, B., and Lacas, F., 2007. "Control of combustion instabilities by local injection of hydrogen”. Proceedings of the Combustion Institute, 31(2), pp. 3207-3214.

[15] Batarseh, F., Gnirß, M., Roisman, I., and Tropea, C., 2009. "Fluctuations of a spray generated by an airblast atomizer". Experiments in Fluids, 46(6), pp. 1081-1091.

[16] Barbosa, S., Scouflaire, P., and Ducruix, S., 2009. "Time resolved flowfield, flame structure and acoustic characterization of a staged multi-injection burner". Proceedings of the Combustion Institute, 32(2), pp. 2965 - 2972.

[17] Barbosa, S., 2008. "Etude expérimentale d'un injecteur multipoint étagé". PhD thesis, Ecole Centrale Paris.

[18] Galley, D., Ducruix, S., Lacas, F., and Veynante, D., 2011. "Mixing and stabilization study of a partially premixed swirling flame using laser induced fluorescence". Combustion and Flame, 158, pp. 155-171.

[19] Higgins, B., McQuay, M., Lacas, F., Rolon, J., Darabiha, N., and Candel, S., 2001. "Systematic measurements of OH chemiluminescence for fuel-lean, high-pressure, premixed laminar flame". Fuel, 80, pp. 67-74.

[20] Lauer, M., and Sattelmayer, T., 2010. "On the adequacy of chemiluminescence as a measure for heat release in turbulent flames with mixture gradients”. Journal of Engineering for Gas Turbines and Power, 132(6), p. 061502.

[21] Lauer, M., Zellhuber, M., Sattelmayer, T., and Aul, C. J., 2011. "Determination of the heat release distribution in turbulent flames by a model based correction of $\mathrm{OH}^{*}$ chemiluminescence”. Journal of Engineering for Gas Turbines and Power, 133(12), p. 121501.

[22] Providakis, T., Zimmer, L., Scouflaire, P., and Ducruix, S., 2012. "Characterization of the coherent structures in swirling flames stabilized in a two-staged multi-injection burner: influence of the staging factor.". Comptes Rendus Mecanique(accepted for publication).

[23] Providakis, T., Scouflaire, P., Zimmer, L., and Ducruix, S., 2010. “Time-resolved PIV measurements applied to a non-reactive dodecane-air mixture in a two-staged multi-injection burner". 15th Int Symp on Applications of Laser Techniques to Fluid Mechanics, July.

[24] Providakis, T., Zimmer, L., Scouflaire, P., and Ducruix, S., 2011. "Effect of fuel distribution on spray dynamics in a two-staged multi-injection burner". Proceedings of ASME Turbo Expo (GT2011-46519), Vancouver, Canada.

[25] Rachner, M., Becker, J., Hassa, C., and Doerr, T., 2002. "Modelling of the atomization of a plain liquid fuel jet in crossflow at gas turbine conditions". Aerospace Science and Technology, 6(7), pp. 495 - 506.

[26] Boxx, I., Stöhr, M., Carter, C., and Meier, W., 2010. "Temporally resolved planar measurements of transient phenomena in a partially pre-mixed swirl flame in a gas turbine model combustor”. Combustion and Flame, 157(8), pp. 1510 1525.

[27] Steinberg, A. M., Boxx, I., Stöhr, M., Carter, C. D., and Meier, W., 2010. "Flow-flame interactions causing acoustically coupled heat release fluctuations in a thermo-acoustically unstable gas turbine model combustor". Combustion and Flame, 157(12), 12, pp. 2250-2266.

[28] Moeck, J. P., Bourgouin, J.-F., Durox, D., Schuller, T., and Candel, S., 2012. "Nonlinear interaction between a precessing vortex core and acoustic oscillations in a turbulent swirling flame". Combustion and Flame, 159(8), pp. 2650 -2668 .

[29] Sujith, R. I., Waldherr, G. A., Jagoda, J. I., and Zinn, B., 1997. "An experimental investigation of the behavior of droplets in axial acoustic fields". Journal of Vibration and Acoustics, 119, pp. 285-292.

[30] Sujith, R. I., 2005. "An experimental investigation of interaction of sprays with acoustic fields". Experiments in Fluids, 38, pp. 576-587. 10.1007/s00348-004-0912-1.

[31] Eckstein, J., Freitag, E., Hirsch, C., Sattelmayer, T., Von der Bank, R., and Schilling, T., 2005. "Forced low-frequency 
spray characteristics of a generic airblast swirl diffusion burner". Journal of Engineering for Gas Turbines and Power, 127(301).

[32] Sujith, R. I., 2003. “An experimental investigation of jets in a strong acoustic field”. AIAA(2003-1276).

[33] Haile, E., Delabroy, O., Lacas, F., Veynante, D., and Candel, S., 1996. "Structure of an acoustically forced turbulent spray flame". In Twenty-Sixth Symposium (International) on Combustion, The Combustion Institute, pp. $1663-1670$.

[34] Gajan, P., Strzelecki, B., Platet, B., Lecourt, R., and Giuliani, F., 2007. "Investigation of spray behavior downstream of an aeroengine injector with acoustic excitation”. Journal of Propulsion and Power, 23(2), March-April, pp. $390-397$.

[35] Lacour, C., Durox, D., Ducruix, S., and Massot, M., 2011. "Interaction of a polydisperse spray with vortices". Experiments in Fluids, 51(2), pp. 295-311.

[36] Birbaud, A. L., Durox, D., Ducruix, S., and Candel, S., 2007. "Dynamics of free jets submitted to upstream acoustic modulations". Physics of Fluids, 19(1), p. 013602. 